\title{
$\therefore$ Assessment of Patient's Quality of Life with Oral Cancer Treatment
}

\section{IJCRR}

Section: Healthcare

ISI Impact Factor

(2019-20): 1.628

IC Value (2019): 90.81

$\operatorname{SJIF}(2020)=7.893$

\section{Arunkumar J1, Dushyant Koul²}

'Professor \& Head, Department of Oral \& Maxillofacial Surgery, Vinayaka Missions Sankarachariyar Dental College, Salem, Tamilnadu, lndia; ${ }^{2}$ PG student, Department of Oral \& Maxillofacial Surgery, Vinayaka Missions Sankarachariyar Dental College, Salem, Tamilnadu, India.

\section{ABSTRACT}

Introduction: Oral cancer is the 2nd most common cancer of the body. Oral cancer can be treated surgically, with chemotherapy or with irradiation. The goal of cancer therapy may be described as the achievement of a disease-free physical state of health.

Objectives: To evaluate the oral functions in patients after the treatment for oral cancer.

Methods: Study includes twenty-three patients of both sexes who had undergone surgery alone or surgery with radiotherapy. In that twenty patients had squamous cell carcinoma of the oral cavity and three patients had verrucous carcinoma. Quality of life was assessed using a questionnaire. Impairment of speech, chewing swallowing, degree of pain and appearance was assessed in all the patients.

Result: The mean age was 58.26 years. The most affected site in the oral cavity was mandible (52\%). There was Grade-I $73.9 \%$ of patients had no pain, Grade-II had $21.7 \%$ mild pain and Grade-III had $4.35 \%$ moderate pain. $60 \%$ had minor changes after the treatment. The majority had improvement in swallowing after treatment (69.5\%). II 52.1\% can chew with mild difficulty. $56.5 \%$ had mild difficulty speech.

Conclusion: Patients receiving surgery and radiotherapy have more significant impairment in chewing and swallowing..

Key Words: Oral cancer, Quality of life, Questionnaire study, Chemotherapy

\section{INTRODUCTION}

Oral cancer is the second most common cancer of the body. Annually, more than 640,000 patients worldwide are diagnosed with primary cancer and approximately 350,000 die of this disease. Approximately 3,00,000 people are affected by cancer per year in India. ${ }^{1}$ Cancer of the head and neck affects most fundamental life function including airway, digestion, chewing, swallowing and speech. ${ }^{2}$ Apart from physical problems, Cancer patients are also affected by psychosocial problems like anxiety, depression, loss of self-esteem and uncertainty about the future. ${ }^{2}$ Oral cancers can be treated surgically, with irradiation, chemotherapy, or with any of the above combination. Conversely, the survival duration itself is not a satisfactory measure of success. ${ }^{3}$ Quality-of-life (QL) is a comparatively new idea in head and neck oncology to measure the quality of life. ${ }^{4,5}$

Facial disfigurement can cause huge aesthetic harms for the patient. Furthermore, side-effects of radiotherapy, such as destruction of the salivary glands, affecting xerostomia extending to the whole of the survival time. Xerostomia can be related to oral infections, pain, dental caries, and uneasiness. Default after surgical procedure and the side-effects of radiation reduces the quality of life. The goal of cancer therapy may be described as the achievement of a diseasefree physical state of health and an acceptable quality of life after treatment. ${ }^{5}$ Advances in cancer treatment have brought about the significant increase in survival of a patient with malignancies. ${ }^{5,6}$

The quality of survival after treatment for head and neck cancer patients is an important consideration for evaluation of treatment and rehabilitation outcome. ${ }^{7}$ To determine the quality of life of cancer patients in general and those with head and neck cancer in particular, several new measures for quality of life have been developed over the past decades. ${ }^{8}$ Basically, quality of life has two fundamental premises. First, it is a multi-dimensional survey incorporating physical, psy-

\section{Corresponding Author:}

Dr. Arunkumar J, Professor and Head, Department of Oral \& Maxillofacial Surgery, Vinayaka Missions Sankarachariyar Dental College Salem, Tamilnadu, India; E-mail: drarunkumar@vmsdc.edu.in

ISSN: 2231-2196 (Print)

Received: 09.09.2020
ISSN: 0975-5241 (Online)

\author{
Revised: 16.10 .2020
}

Accepted: 18.11 .2020
Published: 10.03 .2021 
chological, social, emotional and functional domains. Second, it is subjective and must be self-reporting; according to the patient's own experiences. ${ }^{9}$ Most of the patients with oral cancer are concerned about their oral functions like chewing and swallowing. The effects of these functional limitations on the quality of life, however, may be different due to a variable potential for coping. ${ }^{10}$

In 1947 WHO defined quality of life (QOL) as "an individual's perception of their position in life, in the context of the culture and value systems in their life and concerning their goals, expectations, standards and concern. ${ }^{11-13}$ Quality of life outcome monitoring system for head and neck cancer and reconstructive surgery requires tools that are not only valid but reliably address not only the general health and social factors but also is specific for head and neck cancer. Keeping these in mind Trivandrum quality of life study (TQOLS) was conceived. ${ }^{1}$ QOL used in daily clinical practice to measure the efficiency of treatment. ${ }^{14}$

Through this study, we can evaluate the oral functions in patients after the treatment for oral cancer. This study was done to evaluate the quality of life in patients with oral cancer following treatment. The objectives were to assess swallowing efficiency, Speech efficiency, chewing efficiency, Postoperative Pain, Postoperative appearance acceptance and, coping measures taken by patients following surgery in patients receiving different modalities of treatment for different types of oral malignancies.

\section{MATERIALS AND METHOD}

This study was conducted in the Department of Oral and Maxillofacial surgery, Vinayaka Mission Sankarachariyar Dental College, Salem from 1-4-2014 to 30-11-2014. Twenty-three patients of both sexes who had undergone surgery alone or surgery with radiotherapy during the period from 2008 to 2014 were included.

Demographic profile of each patient was recorded in preformat. Among twenty-three patients, twenty patients had squamous cell carcinoma of the oral cavity and three patients had verrucous carcinoma. Patients were classified according to the site of lesion as those having isolated cheek, lip, tongue, the floor of mouth lesions, maxillary lesions and mandibular lesions. All the patient data that include name, age, sex, location of the tumour, Tumour node metastases (TNM) staging, histopathological diagnosis and treatment were collected. The impairment of speech, chewing swallowing, degree of pain and appearance was assessed in all the patients.

Quality of life was assessed using a questionnaire. All the patients were informed about the study and written consent to be part of the study was taken from the patients. The questionnaire consists of twenty questions exploring five functional scales (chewing, speech, swallowing, pain and appearance) with ten coping measures.

Inclusion Criteria was patients with carcinomas of cheek, tongue, buccal mucosa, palate, maxilla and mandible, who have undergone surgery alone or surgery with radiotherapy. Exclusion Criteria was patient, not willing to be part of the study.

\section{Functional Assessment}

1) Pain (Using VAS)

a) Do you have pain

b) Do you have mild pain

c) Do you have moderate pain

d) Do you have severe pain

Yes [ ] No [ ]

Yes [ ] No []

Yes [ ] No []

Yes [ ] No []

2) Appearance

a) No change in appearance $\quad$ Yes [ ] No [ ]

b) Minor change in appearance Yes [ ] No [ ]

c) Major change in appearance Yes [ ] No [ ]

d) Appearance significantly disfigured Yes [ ] No [ ]

3) Swallowing

a) Do you have difficulty in swallowing Yes [ ] No [ ]

b) Do you swallow only semi-solid and pureed food Yes [ ] No [ ]

c) Can you swallow liquids Yes [ ] No [ ]

d) Are you unable to swallow any food or liquid because it goes down the wrong way and chokes me

4) Chewing

Yes [ ] No [ ]

a) Do you ever have difficulty in chewing Yes [ ] No [ ]

b) Do you have mild difficulty in chewing hard things like chapatti, parotha, meat, raw vegetable

Yes [ ] No []

c) Do you have severe difficulty in chewing hard things Yes [ ] No [ ]

d) Do you usually eat food that doesn't require chewing or you only take liquids Yes [ ] No [ ]

5) Speech
a) No difficulty in speech
Yes [ ] No [ ]
b) Mild difficulty in speech
Yes [ ] No [ ]
c) Severe difficulty in speech
Yes [ ] No []
d) Incomprehensible speech
Yes [ ] No [ ]

6) Coping

How do you cope with your worries and concerns (rate most frequently used as $1,2,3 \ldots \ldots$ )
a) By talking to others: professional
b) Talking to others: family $\&$ friends
c) By distractive action
d) By trying to accept
e) Optimism
f) By self- confidence - fighting spirit
g) Confidence in medical care
h) Post postponement of worry

[] []

[] []

[] []

[] []

[] []

[] []

[] []

[] [] 
i) Have decided not to worry

j) Turning to religion

\section{Assessment of Quality of Life}

Quality of life was assessed using a questionnaire. The questionnaire consists of twenty questions exploring five functional scales (chewing, speech, swallowing, pain and appearance) with ten coping measures. The chewing, speech, pain, appearance and swallowing scale, was graded as shown in the table below.

1) Pain (Using VAS)

Grade I - Have no pain.

Grade II - There is mild pain

(0 on VAS)

(Ranging from

1-3 on VAS)

Grade III - There is moderate pain

(Ranging from

4-6 on VAS)

Grade IV - There is severe pain

(Ranging from

7-10 on VAS)

\section{2) Appearance}

Score I -No change in appearance

Score II -Minor change in appearance.

Score III -Major change in appearance

Score IV -Appearance significantly disfigured

3) Swallowing

Grade I - Can swallow with ease

Grade II - Cannot swallow certain solid food

Grade III - Can only swallow liquid foods.

Grade IV - Cannot swallow because it "goes down the wrong way and chokes me.

4) Chewing

Grade I - Can chew with ease

Grade II - Can chew with mild difficult

Grade III - Can chew with severe difficulty

Grade IV -Cannot chew.

5) Speech

Score I - No difficulty in speech

Score II - Mild difficulty in speech

Score III - Severe difficulty in speech

Score IV - Incomprehensible

\section{RESULTS}

Table 1 indicates age distribution among a patient. Age ranges from 38 to 75 yrs with mean age was 58.26 years. 13 patients were female and 10 were male out of 23 cancer patients. It is evident from Table 2 that the prevalence of oral cancer is more in female patients as compared to the male patients in this series. It is evident from Table 2 that the most affected site in the oral cavity was mandible. $52 \%$ of patients had a lesion in mandible, $21 \%$ of patients had a lesion in cheek, $8.7 \%$ of patients had a lesion in Lip and Maxilla and $4.3 \%$ of patients had lesion intongue\& floor of the mouth. The type of treatment received by 23 patients: $26 \%$ of the patients had undergone surgery alone, and $73.9 \%$ of the pa- tients had surgery with radiotherapy.

Table 3 show that the severity of pain after the treatment: There was Grade-I $73.9 \%$ of patients had no pain, GradeII had $21.7 \%$ mild pain and Grade-III had $4.35 \%$ moderate pain. Table 4 show the change in appearance after the treatment. There was Score-I 26\% of patients had no change, Score-II $60 \%$ had minor changes and Score-III 13\% had a major change in appearance.

Table 5 shows swallowing impairment in 23 patients. There was grade-I $69.5 \%$ of patients can swallow with ease, gradeII $21.74 \%$ of patients could not swallow certain solid foods and Grade-III $8.7 \%$ of patients were able to take liquid diet only. Table 6 shows the chewing impairment of 23 patients. There was Grade-I 26\% of patients can chew with ease, Grade-II $52.1 \%$ can chew with mild difficulty, Grade-III $17.3 \%$ can chew with severe difficulty, and Grade-IV $4.3 \%$ cannot chew.

Table 7 shows the speech impairments in 23patients. There was Score-I $39.1 \%$ had no difficulty in speech, Score-II $56.5 \%$ had mild difficulty in speech and Score-III $4.3 \%$ had severe difficulty in speech. It's evident from the table 8 that, The most common measure was confidence in the medical care, Followed by self-confidence-fighting spirit, Optimism, tying to accept, talking to others: Family and Friends, decided not to worry, Postponement of worry, talking to others: Professionals, Distractive action and Turning to religion.

\section{DISCUSSION}

Oral cancer is one of the commonest cancer in India. ${ }^{1}$ Oral cancer and its treatment have a significant effect on some of the basic human functions like chewing, swallowing and speech. However, cancer treatment has considerably improved the quality of life. ${ }^{15}$ Head and neck cancer and its treatment can have a reflective outcome on the patient's functional, physical, and emotional comfort, particularly reducing the QOL. QOL can be measured by the supervision of detailed questionnaires to the affected patients. ${ }^{3}$ Quality of life is difficult to quantify accurately and is often subjective. ${ }^{16}$

In our study surgical treatment was planned according to the TNM staging. This study was based on questionnaires and parameters evolved by the TQOLS, which is a QOL evaluator developed in RCC Trivandrum. ${ }^{1}$ The quantifying criteria for pain, appearance, speech chewing and swallowing was evolved based on the degree of pain, change in appearance, difficulty in speech and their ability to chew, swallow different types and consistencies of local food and were awarded grades from 1 to 4 . Ten criteria's of coping were chosen from 34 possible coping techniques. ${ }^{1}$ 


\section{Degree of pain}

In our study pain was recorded by using VAS scale with readings ranging from $0-10$ where ' 0 ' corresponds to no pain and ' 10 ' to the worst pain imaginable. In cancer patients with pain, the sensitivity and validity of this scale have been recognized. ${ }^{17}$ Degree of pain has a great impact on the quality of life. Min et al., Evaluated changes in quality of life of head and neck cancer patients following post-operative radiotherapy and reported that $70 \%$ of the patients had no pain after surgery followed by radiotherapy which correlates with our study results where $73.9 \%$ of patients had no pain. ${ }^{9}$ Klug et al. reported that $21 \%$ of patients had mild pain after the surgeries, which were followed for a period of 2 to 10 years, similar to our study results where $21.7 \%$ of patients had mild pain post-surgery which continued for six months. ${ }^{6}$ The pain was reported in $46.7 \%$ of patients in the study conducted by Pandey Manoj et al. Nils-Claudius Gellrich et al. used DOSAK rehabilitation questionnaire to study the degree of pain following treatment for oral cancer. They reported that pain was present in $10 \%$ of patients in the oral cavity and neck region. $^{7}$

\section{Change in Appearance}

In our study change in appearance has a great effect on the QOL of the treated patients. $\mathrm{Klug}^{6}$ et al. reported that $22 \%$ of patients had no change in appearance and $14 \%$ reported change in appearance that is a major change in appearance which bothered them. Which correlates with our study results where $26 \%$ of patients had no change in appearance. Nils-Claudius Gellrich ${ }^{7}$ et al., used DOSAK rehabilitation questionnaire to study the change in appearance following treatment for oral cancer. They reported that $84 \%$ of the patients had a minor change in appearance and $15 \%$ had a major change in appearances while in our study results $60 \%$ had minor changes in appearance and $13 \%$ had major changes in appearances. Pandey Manoj et al. reported change in appearance was felt by $20 \%$ of the patients but the amount of change was not specified in his study. ${ }^{1}$ Chaturvedi et al., reported that $38 \%$ of the patients had disfigurement of the appearance, this disfigurement interferes with social activity of patients. The study included 50 head and neck cancer patients. ${ }^{16}$

\section{Swallowing Impairment}

In our study swallowing impairment of Grade-I that is patient had no difficulty to swallow and were able to swallow with ease was reported by $69.5 \%$ of patients. Nils - Claudius Gellrich et al., used DOSAK rehabilitation questionnaire to study impairment of swallowing following treatment for oral cancer. ${ }^{17}$ They reported that swallowing impairment was not existent in $24.8 \%$ of patients, small in $19.5 \%$ modest in $25.5 \%$ strong in $17.7 \%$ and heavy in $12.3 \%$ of patients. They emphasize that swallowing might be less influenced by post-treatment measures such as dentures and tongue mobilization than by problems that are directly influenced by surgical treatment and radiotherapy, such as xerostomia and mandibular discontinuity.

Klug et al. reported $23.8 \%$ of patients had difficulty in swallowing liquid, $37.3 \%$ in swallowing pureed food and 56.7 $\%$ in swallowing solid foods. But both the studies did not address the relationship between the site of lesions and swallowing impairment. ${ }^{6}$

\section{Chewing Impairment}

Chewing has a great impact on health-related quality of life. Pandey et al. reported that $46.7 \%$ of the patients had chewing difficulty following treatment for various oral malignancies. The result correlates with our study results. ${ }^{10}$ But they did not address the relationship between the site of lesion and chewing impairment. Patients treated for mandible and maxillary lesion adapt mostly to semi-solid diet, whereas most of the patients treated for isolated cheek and tongue lesions were able to take solid foods. Finlay et al. also reported that patients who had undergone maxillectomy procedures have minimal chewing impairment. ${ }^{18}$

Rogers et al., Compared obturation \& free flap reconstruction in maxillectomy patients. They reported obturator patients are more concerned about their appearance, have more pain and soreness in their mouth and less satisfied with the function. In our study, following maxillectomy, a functional obturator was given, which improved the patients chewing ability. Chaturvedi et al. reported that $36 \%$ of the patients had mild difficulty in chewing which correlates with our study results where $52 \%$ of patients had mild difficulty in chewing. ${ }^{16,19}$

\section{Speech Impairment}

Patients treated for mandible and tongue lesion had difficulty in speech; whereas most of the patients treated for isolated cheek and lip lesions the speech was coherent.

\section{Surgery vs combined (surgery and radiothera- py) treatment modality}

Combined modality treatment appears to have an adverse effect on dietary intake. In our study patients treated with combined modality had maximum chewing and swallowing impairment respectively, whereas least grade of impairment was not present in any patients treated with surgery alone. In our study postoperative radiotherapy was advised to the patients with $\mathrm{T}_{4}$ lesion. After segmental resection of mandible and maxillectomy, reconstruction was done with a stainless steel reconstruction plate and functional obturator respectively. Lip defect was reconstructed with nasolabial flap. Composite cheek defect was reconstructed with pectoralis major myocutaneous flap, the buccal pad of fat, radial forearm flap and 
forehead fasiocutaneous flap. Patients receiving combined modality treatment usually had larger tumours requiring wider surgical excision which may partly explain the differential dietary intake between the two groups. Other factors include xerostomia, loss of taste and loss of appetite which some patients experience the following radiotherapy

\section{Coping}

Coping refers to thought and acts used by an individual to manage the internal and external demands of both that tax or exceed his or her psychological resources. The coping is conceived as a dynamic process and depends on the person's environmental transaction. In our study the commonest coping measure used was by confidence in the medical care followed by self-confidence fighting spirit, Optimism, trying to accept, talking to others: family and friends, decided not to worry, postponement of worry, talking to others: professionals, by distractive action and the last coping measure opted, was by turning to religion. This is invariance to the study conducted by Pandey et al., where the majority of patients turn to religion and also. Scharloo et al., suggest that restructuring negative pretreatment illness perceptions may help patients to cope more better. ${ }^{20}$

The study while being broadly indicative of the quality of life, one needs to remember that quality of life is objective and it is modified by extraneous factors. In our study only six criteria have been evaluated whereas a comprehensive quality of life should assess other significant afflictions like deformity, restriction in shoulder movement, absence of taste, dry mouth, limitation in mouth opening, social acceptance etc. similarly coping evaluated is isolation is not fully meaningful feedback. Coping needs to be assessed in conjunction with concerns. However, concerns and coping can only be evaluated by a prospective study. The subject holds out many exciting possibilities for understanding and improving the quality of life in cancer patients. A large multicentric prospective study will throw more light on these issues.

\section{CONCLUSION}

Patients receiving surgery and radiotherapy have more significant impairment in chewing and swallowing. This may be attributed to xerostomia and post-radiotherapy fibrosis. A review of coping techniques reveals that the methods used most often for coping were confidence in medical care. Surprisingly spirituality was not a favourite coping strategy.

\section{Conflict of Interest: Nil}

\section{Source of Funding: Self}

Authors contribution

1. Dr. Arunkumar J *- Investigation, Data collection

2. Dr. Dushyant Koul- Analysis, Manuscript writing

\section{REFERENCES}

1. Pandey M, Latha PT, Mathew A, Ramdas K, Chaturvedi SK. Concerns and coping strategies in patients with oral cancer. Ind J Surg 2003;65(6):496-499.

2. Rogers SN, Fisher SE, Woolgar JA. A review of the quality of life assessment in oral cancer. Int J Or Maxill Surg 2002;199(28):99117.

3. Kazi R, Sayed S, Dwivedi RC: Clinical importance of quality of life measures in head and neck cancer. Ind J Cancer 21010;47:237-238.

4. Morton RP, Izzard ME: Quality of life outcomes in head and neck cancer patients. World J Surg 2003;27:884-889.

5. Schliephak E H, Karsten Rüffert, Thomas Schneller. Prospective study of the quality of life of cancer patients after intra oral tumor surgery. J Oral Maxill Surg 1996;54:664-669.

6. Klug C, Neuburg J, Glaser C, Schwarz B, Kermer C, et al. Quality of life 2-10 years after combined treatment for advanced oral and oropharyngeal cancer. Inter J Oral Maxill Surg 2002;31:664669.

7. Gellrich N, Schramm A, Böckmann R, Kugler J. Follow up in patients with oral cancer. J Oral Maxill Surg 2002;60:380-386.

8. Schwartz CE, Daltroy LH, Brandt U, Friedman R, Stolbach L. A psychometric analysis of the mental adjustment to cancer scale. Psychol Med 1992; 22:203-210.

9. Min-Fang, Chien CY, Kuo SC, Chiu HC, Wang CJ. Changes in quality of life of head and neck cancer patients following postoperative radiotherapy. Acta Oncol 2004; 432(6):571-578.

10. Boin IS, Briancon S. Impact of cancer occurrence on health related quality of life: A longitudinal pre- post assessment. Heal Qual Life Outcomes 2004;2:4:1-11.

11. Torres-Carranza E, Infante-Cosso P, Hernández-Guisado JM, Hens Aumente E, Gutiérrez-Pérez JL: Assessment of quality of life in oral cancer. Med Oral Patol Oral Cir Buccal 2008;13(11): E735-741.

12. Kazi R, Johnson C, Prasad V, De Cordova J, Venkitaraman R, et al. Quality of life outcome measures following partial glossectomy: using the UW- QOL scale. J Canc Res Ther 2008;4: 116-120.

13. Sayed SI, Elmiyeh B, Rhys-Evans P, Syrigos KN, Nutting CM, et al. Quality of life and outcomes research in head and neck cancer: A review of the state of the discipline and likely future directions. Canc Treat Rev 2009;35(5): 397-405.

14. Lopez JH, Mayordomo AR, Rosado RL, Fernandez CIS, Gallana S. Quality of life in long-term oral cancer survivors: A comparison with Spanish general population norms. J Oral Maxill Surg 2009;67:1607-1614.

15. Schiliephake H. Prospective evaluation of the quality of life after oncologic surgery for oral cancer. Int J Or Maxill Sur 2002;31:427-433.

16. Chaturvedi SK, Chandra PS. Concerns, coping and quality of life in head and neck cancer patients. Supp Care Can 1996;4:186-190.

17. Kandasamy A, Chaturvedi SK, Desai G. Spirituality, distress, depression, anxiety and quality of life in patients with advanced cancer. Ind J Can 2011;48(1):55-59.

18. Finlay PM, Dawson F, Robertson AG, Soutar DS. An evaluation of functional outcome after surgery and radiotherapy for intraoral cancer. Br J Oral Maxill Surg 1992;30(1):14-17.

19. Rogers SN, Derek Lowe, James S Brown. Health-related quality of life after maxillectomy: A comparison between prosthetic obturation and free flap. J Oral Maxill Surg 2003; 61:174-181.

20. Scharloo M, Baatenburg De Jong R J.Quality of life and illness perceptions in patients with recently diagnosed head and neck cancer. J Hea Nec 2005;27(10):857-863. 
Table 1: Age and gender Distribution

\begin{tabular}{lcc} 
Age in yrs & $\begin{array}{c}\text { Mean Age Distribution } \\
\text { Frequency }\end{array}$ & Percent \\
38 & 1 & 4.35 \\
40 & 2 & 8.70 \\
45 & 2 & 8.70 \\
50 & 1 & 4.35 \\
55 & 1 & 4.35 \\
56 & 2 & 8.70 \\
59 & 1 & 4.35 \\
60 & 2 & 8.70 \\
61 & 2 & 8.70 \\
65 & 3 & 13.04 \\
66 & 1 & 4.35 \\
67 & 1 & 4.35 \\
68 & 1 & 4.35 \\
70 & 1 & 4.35 \\
73 & 1 & 4.35 \\
75 & 1 & 4.35 \\
Total & 23 & 100.00 \\
& Gender distribution & \\
Sex & Frequency & Percent \\
Male & 10 & 43.48 \\
Female & 13 & 56.52 \\
Total & 23 & 100.00 \\
\hline & &
\end{tabular}

Table 2: Site of Lesion and type of procedure

\begin{tabular}{lcc} 
1. Site of lesion & & \\
Site Lesion & Frequency & Percent \\
Tongue & 1 & 4.35 \\
Cheek & 5 & 21.74 \\
Lip & 2 & 8.70 \\
Floor of mouth & 1 & 4.35 \\
Maxilla & 2 & 8.70 \\
Mandible & 12 & 52.17 \\
Total & 23 & 100.00 \\
2. Type of Procedure & & \\
Procedure & Frequency & Percent \\
Surgery & 6 & 26.09 \\
Surgery+RT & 17 & 73.91 \\
Total & 23 & 100.00 \\
\hline
\end{tabular}

Table 7: Speech Impairment

\begin{tabular}{lcc} 
Speech & Frequency & Percent \\
No difficulty in speech & 9 & 39.13 \\
Mild difficulty in speech & 13 & 56.52 \\
Severe difficulty in speech & 1 & 4.35 \\
Total & 23 & 100.00 \\
\hline
\end{tabular}

Table 8: Coping

\section{Coping}

Mean Rank

By talking to others: Professionals

7.04

Talking to others :Family and Friends

5.65

By distractive action

8.70

By trying to accept

4.54

Optimism

3.17

By self-confidence, fighting spirit

2.91

Confidence in medical care

1.22

Postponement of worry

6.52

Have decided not to worry

$5 \cdot 59$

Turning to religion 